\title{
Prevalence and phylogenetic characterizations of Enterovirus D68 strains among respiratory infection cases in Beijing, 2018-2019
}

\author{
Aihua $\mathrm{Li}^{1}$, Tiegang zhang ${ }^{2}$, Cheng Gong ${ }^{3}$, Maozhong $\mathrm{Li}^{2}$, and Fang Huang ${ }^{3}$ \\ ${ }^{1}$ Beijing Center for Disease Prevention and Control \\ ${ }^{2}$ Affiliation not available \\ ${ }^{3}$ Beijing Center for Disease Prevention and Control, Beijing Preventive Medicine Research \\ Center
}

August 5, 2020

\begin{abstract}
Backdround: Enteroviruse-D68 (EV-D68) has been endemic in Beijing for some years. This study analyz the prevalence and phylogenetic characterizations of EV-D68 in Beijing during 2018 to 2019. Methods: The clinical specimens were collected from respiratory infection cases of 30 sentinel hospitals in Beijing and subjected to EV and EV-D68 detection by real-time PCR. Results: Of 15, 645 respiratory infection cases, 467(2.98\%) cases were enterovirus positive and 14(0.09\%) were EV-D68 positive. The detection rates of EV-D68 were $0.11 \%(9 / 7,837)$ in $2018,0.06 \%(5 / 7,805)$ in 2019 , respectively. Of these EV-D68 infection patients, 7 cases were aged younger than 18 years, 3 were aged 18 to 60 years and 4 aged over 60 years. 4 cases (28.6\%) were diagnosed as upper respiratory tract infection and $10(71.4 \%)$ as pneumonia, including one with severe pnuemonia. The main symptoms for EV-D68 patients were fever (10/14,71.4\%) and cough (9/14,64.3\%). 7 cases were detected in summer and the rest cases in the autumn. The phylogenetic analysis suggested that EV-D68 strains from 2018 belonged to a major subclade B3 and a minor subclade D3, while all strains in 2019 were clustered into subclade D3. These subclade B3 or subclade D3 strains were located in a separated evolutionary branches,respectively. Conclusions: Our research further elucidate the continuous molecular evolution and genetic variability of EV-D68 occured in Beijing, China.
\end{abstract}

\section{Hosted file}

Prevalence and phylogenetic characterizations of Enterovirus D68 strains among respiratory infection ca available at https://authorea.com/users/348837/articles/474037-prevalence-and-phylogeneticcharacterizations-of-enterovirus-d68-strains-among-respiratory-infection-cases-inbeijing-2018-2019 